\title{
Ion Association by Time-of-Flight Mass Spectrometry: A Study of V-P-O Catalysts
}

\author{
J. K. Schoer, ${ }^{*}$ R. S. Houk, R. J. Conzemius, and G. L. Schrader \\ Ames Laboratory-U.S. Department of Energy, Departments of Chemistry and Chemical Engineering, \\ Iowa State University, Ames, Iowa, USA
}

The oxidation catalysts $\left(\mathrm{VO}_{2}\right) \mathrm{P}_{2} \mathrm{O}_{7}$ and $\beta$-VOPO 4 are studied by laser desorption time-offlight mass spectrometry. Mass spectra of both positive and negative ions are reported for these compounds. Individual scans are tested for ion association, that is, the consistent appearance of a given pair of ions in many scans. The structurally simpler $\beta-\mathrm{VOPO}_{4}$ shows fewer associations than either (VO) ${ }_{2} \mathrm{P}_{2} \mathrm{O}_{7}$ or an ${ }^{18} \mathrm{O}$-substituted analogue of $\beta$-VOPO $\mathrm{VO}_{4}$ even though the overall spectra from the three compounds do not differ greatly. These results indicate that the associations observed between ions may be sensitive to small differences in structure in the original specimen. Thus, the identification of ion association may provide information to supplement the mass spectrum of the sample. (J Am Soc Mass Spectrom 1990, 1, 129-137)

$\mathrm{T}$ ime-of-flight mass spectrometry (TOF/MS) has elicited major research interest in recent years. One interesting aspect of TOF/MS is its ability to record a major fraction of all the ions generated in a pulsed ionization event. Recent advances in instrumentation for data acquisition in TOF/MS permit the counting of individual ions and the recording of ion arrival times for many ionization events at high repetition rates [1, 2]. Previous work has shown that these stored scans can be evaluated for ion association, that is, the consistent appearance of two different ions together in individual scans. The initial work in this area mainly demonstrated the capabilities of the instrumentation and showed that some pairs of ions were associated, whereas others were not [3].

The present work extends this critical evaluation of ion association to the study of two V-P-O selective oxidation catalysts. Both are used in the dominant industrial route for converting $n$-butane to maleic anhydride, which is an important chemical intermediate [4-6]. Two V-P-O phases, $(\mathrm{VO})_{2} \mathrm{P}_{2} \mathrm{O}_{7}$ and $\beta$-VOPO ${ }_{4}$, have attracted much attention because of their high activity and selectivity for producing maleic anhydride. Schematic diagrams of their structures are shown in Figures 1 and 2. Important questions remain regarding the structure of these catalysts and the nature of the active sites, despite their characterization by various spectroscopic techniques [6-11]. Only a few mass spectrometric studies of the V-P-O catalysts have been reported, and most of these studies have addressed mechanistic questions concerning oxygen insertion in the hydrocarbon $[6,11]$. These catalysts were attractive for TOF/MS because their major elements are nearly monoisotopic, so their mass spectra are not complicated by isotope peaks. The two compounds also con-

\footnotetext{
* Present address: Hazelton Environmental Services, 515 Science Drive Madison, WI 53711.

Address reprint requests to R. J. Conzemius, Ames Laboratory, U.S. Department of Energy, Departments of Chemistry and Chemical Engineering, Iowa State University, Ames, IA 50011.
}

tain the same elements and differ only in stoichiometry and in certain structural aspects. If the observed ion associations differ for the two compounds, perhaps useful structural information can be inferred from such studies. Thus, this article is intended mainly as a further evaluation of the information provided by ion association.

\section{Experimental}

\section{Instrumentation}

The LD/TOF/MS used in this study has been described previously [12]. Pertinent features of the device and typical operating conditions are listed in Table 1 . The solid sample was coated onto a gold film sputtered onto a quartz plate. The accelerating potential was applied to the gold film via the clamping arrangement used to support the sample holder. The laser irradiated the sample from the back side through the quartz into the gold film, with the sample on the other side of the film. The desorbed ions traversed a linear 1.2-m-long flight tube and were detected by a microchannel plate electron multiplier [13]. The arrival times of up to eight ions per laser pulse were measured by a time-to-digital converter (TDC) and stored in a computer. The dead time was $4 \mathrm{~ns}$, and the time resolution was $1 \mathrm{~ns}$ [2]. The collection of observed arrival times from each laser pulse was referred to as a scan. Mass spectra were obtained by summing 1000 individual scans of the mass range. Additionally, the stored arrival times from each scan were examined for associations between pairs of ions and for multiplicity, as described below.

\section{Sample Preparation}

The quartz plates $(0.75 \times 0.75 \times 0.16 \mathrm{~cm})$ were cut and were then cleaned with a series of solvent washes (soap, hot tap water, deionized distilled water, 1,1,2-trichloroethane, acetone, and methanol). Solvent 


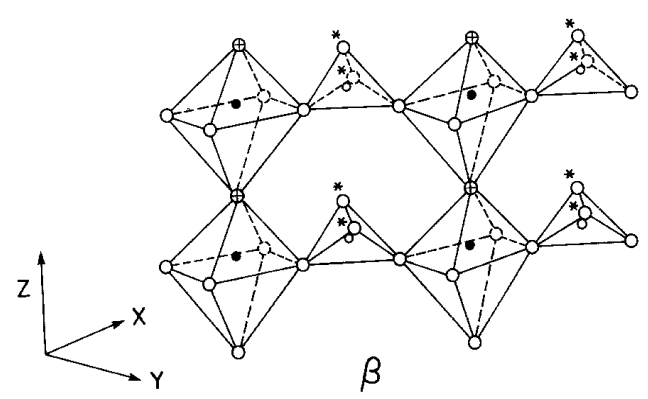

Figure 1. Coordination polyhedra around $\mathrm{V}$ and $\mathrm{P}$ in $\beta-\mathrm{VOPO}_{4}$ : $(\bullet) \mathrm{V} ;(\bigcirc)$ oxygen; $(\oplus)$ vanadyl oxygen. Note that the polyhedra are distorted and the vanadium atoms are not in the centers of the octahedra. In ${ }^{18} \mathrm{O}$-labeled $\beta$-VOPO ${ }_{4}$, the $\mathrm{O}$ sites denoted with asterisks are labeled preferentially [7]. Reproduced from ref 8 with permission.

residue was removed by drying the plates in a clean oven at $150{ }^{\circ} \mathrm{C}$ for $24 \mathrm{~h}$.

A thin film $(\sim 50 \mathrm{~nm})$ of gold was sputtered onto the quartz plates, which were then placed in a clean oven at $150^{\circ} \mathrm{C}$ for $24-48 \mathrm{~h}$ to remove volatile organic contaminants and water from the gold. The layer of gold provided an accelerating potential to the ions, helped minimize charge buildup in the sample layer, and served as a medium to absorb and disperse the laser energy. This promoted the prompt emission of ions with more uniform kinetic energies, both crucial factors for obtaining good resolution in linear, electrostatic TOF/MS. The 50-nm film was the best of several thicknesses tested, as it provided stable flight times, good resolution, ions of a wide range of masses, and little observed delayed emission (i.e., "tailing" of a peak on its high-mass side). Thicker films (100 nm or greater) yielded shorter, more variable flight times, decreased ion production, a higher abundance of heavy ions, and poorer resolution. Decreasing the thickness of the gold film caused longer ion flight times (by up to $50 \mathrm{~ns}$ ), a higher abundance of low-mass ions, increased ion production, more significant delayed emission, and broader peaks.

$\beta-\mathrm{VOPO}_{4}$ and $(\mathrm{VO})_{2} \mathrm{P}_{2} \mathrm{O}_{7}$ were synthesized by using solid-state reactions as described by Moser and
Schrader [14]. The ${ }^{18} \mathrm{O}$-substituted $\beta$-VOPO ${ }_{4}$ was prepared by placing $0.5 \mathrm{~g}$ of $(\mathrm{VO})_{2} \mathrm{P}_{2} \mathrm{O}_{7}$ in a $6 \mathrm{~mm}$ i.d. $\times 46$ $\mathrm{cm}$ Pyrex tube. The tube was evacuated to $10^{-4}$ torr and backfilled with a stoichiometric amount of ${ }^{18} \mathrm{O}_{2}$ (97.6\% purity, MSD Isotopes). It was then heated at $550{ }^{\circ} \mathrm{C}$ for $24 \mathrm{~h}$. Raman spectroscopic studies showed that roughly $50 \%$ of the oxygen sites were substituted with ${ }^{18} \mathrm{O}$. As can be seen from Figure 1 , the ${ }^{18} \mathrm{O}$ went preferentially into the $\mathrm{O}$ sites that were in the $\mathrm{PO}_{4}$ tetrahedra but were not shared with the $\mathrm{VO}_{6}$ octahedra [7].

After synthesis, the samples were dissolved in acetone (Fisher reagent grade). A volume of solution containing $2-4 \mathrm{nmol}$ of sample was deposited by pipet onto the gold-coated quartz plate. The acetone was allowed to evaporate, and the sample plate was then heated to $60^{\circ} \mathrm{C}$ at $10^{-3}$ torr overnight to remove residual solvent. Samples were stored in a $\mathrm{P}_{2} \mathrm{O}_{5}$-charged desiccator for up to one week. Old samples were discarded after one week to avoid complications from absorption of water.

\section{Operating Parameters and Calibration}

The TDC could record the arrival of only up to eight ions in a given scan. Ions that struck the detector after the first eight are not recorded. Several steps were taken to avoid premature filling of the TDC and the loss of late-arriving ions at higher mass. The laser energy was kept at the threshold of ion production by using a combination of neutral density filters and iris settings (Table 1). Slight changes in laser energy caused significant changes in ion intensities, in agreement with other observations of ion production near threshold in LD [12]. Minimizing the laser power also attenuated peak tailing caused by delayed emission, particularly for $\mathrm{Na}^{+}, \mathrm{K}^{+}$, and $\mathrm{Ag}^{+}$[12].

The data system provided programmable time gates or windows, which served to blind the TDC to ions in preselected regions. Thus, cluttered spectral regions or intense ions that were not characteristic of the V-P$\mathrm{O}$ sample (e.g., $\mathrm{K}^{+}, \mathrm{Na}^{+}$, or $\mathrm{Au}^{+}$) could be ignored to avoid premature filling of the TDC and to facilitate observation of ions from the V-P-O compound [2].
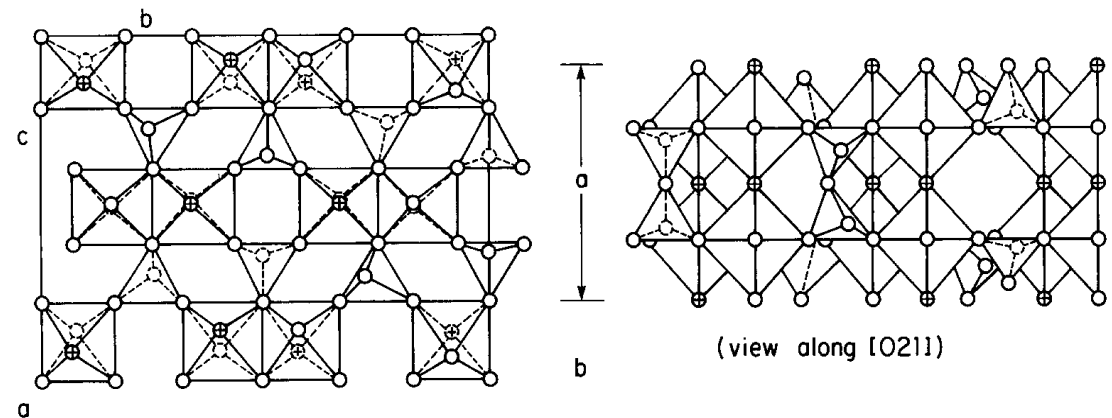

Figure 2. Crystal structure of $(\mathrm{VO})_{2} \mathrm{P}_{2} \mathrm{O}_{7}$. (a) (100) plane; (b) (001) plane. Vanadium and phosphorus atoms are not shown; otherwise same legend as Figure 1. Reproduced from ref. 8 with permission. 
Table 1. Instrumental facilities [12]

\begin{tabular}{|c|c|c|}
\hline \multirow{2}{*}{$\begin{array}{l}\text { Component (Source) } \\
\begin{array}{l}\text { Nitrogen laser (Photochemical Research Associates Inc., } \\
\text { Model LN 120) }\end{array}\end{array}$} & \multicolumn{2}{|c|}{ Operating materials, parameters, or dimensions } \\
\hline & $\begin{array}{l}\text { Wavelength } \\
\text { Pulse width, nominal } \\
\text { Repetition rate } \\
\text { Energy/pulse } \\
\text { Lens focal length } \\
\text { Iris diameter }\end{array}$ & $\begin{array}{l}337.1 \mathrm{~nm} \\
300 \mathrm{ps} \\
16 \mathrm{~Hz} \\
10-70 \mu \mathrm{J} \\
2.5 \mathrm{~cm} \\
0.75-1.5 \mathrm{~mm}\end{array}$ \\
\hline \multirow[t]{2}{*}{$\begin{array}{l}\text { Neutral density filters (Melles Griot, Inc.) } \\
\text { Ion source (Ames Laboratory Construction [12]) }\end{array}$} & $\begin{array}{l}\text { Total optical density } \\
\text { Accelerating voltage }\end{array}$ & $\begin{array}{l}0.3 \\
+4500 \vee \text { for positive ions; } \\
-4500 \vee \text { for negative ions }\end{array}$ \\
\hline & $\begin{array}{l}\text { Accelerating region } \\
\text { Gold film thickness } \\
\text { Quartz plate thickness }\end{array}$ & $\begin{array}{l}3 \mathrm{~mm} \text { long } \\
\sim 50 \mathrm{~nm} \text { thick } \\
1.6 \mathrm{~mm} \text { thick }\end{array}$ \\
\hline $\begin{array}{l}\text { TOF mass analyzer and ion optics } \\
\text { (Ames Laboratory Construction [12]) }\end{array}$ & $\begin{array}{l}\text { Flight path length } \\
\text { Flight path diameter } \\
\text { Operating pressure } \\
\text { Deflection plate voltage } \\
\text { Einzel lens voltage }\end{array}$ & $\begin{array}{l}1.2 \mathrm{~m} \\
15 \mathrm{~cm} \\
-1 \times 10^{-7} \text { torr } \\
0 \mathrm{~V} \\
0 \mathrm{~V}\end{array}$ \\
\hline $\begin{array}{l}\text { Vacuum pumps (Turbomolecular pumps: } \\
\text { Leybold-Heraeus Vacuum Product, Inc., } \\
\text { Models TMP } 150 \text { and TMP } 300 \text { ) }\end{array}$ & Pumping speeds & 150 and $350 \mathrm{~L} / \mathrm{s}$ \\
\hline $\begin{array}{l}\text { Ion detector (Microchannel plates: } \\
\text { Varian, Model VUW8946; } \\
\text { Housing: Ames Laboratory Construction [13]) }\end{array}$ & Voltage bias on the three stages & $-1800 \mathrm{~V},-900 \mathrm{~V},-100 \mathrm{~V}$ \\
\hline $\begin{array}{l}\text { Data acquisition system (Time-to-digital converter: } \\
\text { LeCroy Research Systems Corporation, Model 4208; } \\
\text { Computer: Hewlett-Packard, Model Vectra } 45\end{array}$ & $\begin{array}{l}\text { Number of scans collected } \\
\text { per experiment } \\
\text { Time resolution of spectra }\end{array}$ & $\begin{array}{l}1000 \\
4 \mathrm{~ns}\end{array}$ \\
\hline
\end{tabular}

For all experiments, 1000 pulses of the laser were used as a reasonable compromise between total ion production and sample lifetime. The ensemble of results from 1000 scans of the mass range represented a single experiment. The sample was moved frequently (relative to the stationary laser, ion optics, and flight tube) between experiments to provide a fresh region for desorption.

The mass scale was calibrated from the measured arrival time of ions, as described previously [12]. For spectra of positive ions, the arrival times for ${ }^{23} \mathrm{Na}^{+}$ and ${ }^{197} \mathrm{Au}^{+}$were used for calibration. Initial studies of negative ions employed measurement of the flight times of ${ }^{79} \mathrm{Br}^{-}$and ${ }^{197} \mathrm{Au}^{-}$from a $\mathrm{NaBr}$ film. Later, ${ }^{31} \mathrm{P}^{16} \mathrm{O}_{3}^{-}$and $\mathrm{Au}^{-}$from the V-P-O films were used. Flight times were quite stable during the six-month period encompassing these experiments. For example, the flight time for the center of the $\mathrm{Au}^{+}$peak was $18,284 \mathrm{~ns}$ with a range of $\pm 8 \mathrm{~ns}$. The flight time for the center of the $\mathrm{Au}^{-}$peak was $18,344 \pm 16$ ns. Negative ions were slowed somewhat as they reached the field gradient near the detector, resulting in slightly longer flight times than for positive ions of nearly the same $m / z$ values.

\section{Data Treatment}

As in the work of Huang et al. [3], identification of ionpair association was based on calculations using the $\chi^{2}$ and $\gamma$ (correlation coefficient) functions. These statistical tests were applied to each of the 1000 scans to de- termine if a given pair of ions were observed together consistently in the same scan above a certain confidence level. Ion-pair associations were determined to occur in a given experiment if $\chi^{2} \geq 12.1$ (99.95\% confidence level) and if $\gamma \geq 0.070$ ( $85 \%$ confidence level).

To determine if an ion-pair association occurred for a compound, the $\chi^{2}$ and $\gamma$ values for all experiments with the same compound and set of parameters were tabulated. Typically, 35-40 experiments were performed for each compound. For a particular pair of ions, association was considered to occur if $\chi^{2}$ and $\gamma$ were above the critical values in at least $80 \%$ of the experiments. The observed associations were compiled in figures called ion-association maps. In some cases, weaker associations (i.e., association observed in $60-80 \%$ of the experiments) or unusually strong associations (high values of $\gamma$ and $\chi^{2}$ ) were seen. These different grades of association were tabulated in ref $\mathbf{1 5}$ but are not considered in the discussion below.

\section{Results and Discussion}

\section{Background Spectra}

Positive ion spectra of blank gold films were very similar to those seen earlier [12]. The major ions were $\mathrm{Au}^{+}$, $\mathrm{Au}_{n}^{+}, \mathrm{K}^{+}$, and $\mathrm{Na}^{+}$. Some $\mathrm{Ag}^{+}$was also commonly seen. The major negative ions were identified as $\mathrm{CN}^{-}$, $\mathrm{Au}^{-}$, and $\mathrm{HOCN}^{-}$. In both modes, most $\mathrm{m} / \mathrm{z}$ values had no counts, but one or two counts were seen occasionally. Thus, the background was low except at $\mathrm{m} / \mathrm{z}$ values that coincided with an actual background peak. 


\section{Mass Spectra of V-P-O Compounds}

Mass spectra of positive and negative ions are shown in Figures 3-5. Several of the more prominent ions are labeled by numbers on the plotted spectra. All ions observed are identified by number in Table 2 . Blank regions in the spectra generally represent $m / z$ positions of background ions that were "gated out" or ignored by the data system to prevent overfilling the TDC. The spectra are plotted directly as the number of ion counts recorded at intervals of 1 ns without smoothing or filtering. Thus, the background appears greater than the actual case, and the peak heights merely show the total number of counts for each species. The peaks were approximately $10 \mathrm{~ns}$ across at the base and were roughly Gaussian as reported previously [12]. The peak shapes and widths are not depicted accurately in the spectra shown. Resolution was at least 1000 in the positive ion mode and at least 500 in the negative ion mode.

Comparison of Figures $3 \mathrm{a}$ and $3 \mathrm{~b}$ indicates that the positive ion spectra from (VO) ${ }_{2} \mathrm{P}_{2} \mathrm{O}_{7}$ were qualitatively similar to those from $\beta-\mathrm{VOPO}$. A few more high-mass ions (i.e., above ${ }^{197} \mathrm{Au}^{+}$) were seen from $(\mathrm{VO})_{2} \mathrm{P}_{2} \mathrm{O}_{7}$. The $\mathrm{Au}^{+}$ion was gated out on Figure $3 \mathrm{c}$, which shows that isotopic substitution of $\beta-\mathrm{VOPO}_{4}$ led to the expected isotope peaks but also caused formation of more positive ions at high mass (e.g., $\mathrm{VP}_{2} \mathrm{O}_{8}^{+}$, no. 23 in Figure $3 \mathrm{c}$ ) compared to unsubstituted $\beta-\mathrm{VOPO}_{4}$. Qualitatively similar effects were seen for negative ions (Figures 4 and 5); that is, more ions were seen from (VO) ${ }_{2} \mathrm{P}_{2} \mathrm{O}_{7}$ and ${ }^{18} \mathrm{O}$-substituted $\beta$-VOPO ${ }_{4}$ than from unsubstituted $\beta$-VOPO ${ }_{4}$.

The ion formulas in Table 2 were assigned from the known structures of the compounds (Figures 1 and 2). As in previous work with $\mathrm{CsI}$ and $\mathrm{YBa}_{2} \mathrm{Cu}_{3} \mathrm{O}_{7}[3,12]$, the ions observed from the V-P-O compounds were generally assigned to aggregates of atoms that were adjacent to the original solid. These aggregates were presumably ejected into the gas phase during laser bombardment. The ion $\mathrm{P}_{2} \mathrm{O}_{4}^{-}$from $\beta-\mathrm{VOPO}_{4}$ was an exception to this generalization. The ion abundances were low. No ions at all were observed in roughly half the scans, which indicated that the laser power was indeed near the threshold for ion production.

The potential problems from overrunning the TDC are depicted in Figure 5, which compares two spectra from ${ }^{18} \mathrm{O}$-substituted $\beta$-VOPO ${ }_{4}$. No gates were used in Figure 5a; hence few ions above 13,000 ns (roughly $m / z=100$ ) were detected. The same sample was analyzed with a single gate that allowed the TDC to ignore ions below $12,000 \mathrm{~ns}(m / z \approx 79)$. As shown in Figure $5 b$, various heavy ions were then observed. Apparently, lower mass negative ions were produced in sufficient abundance from this sample to readily fill the TDC and cause a misleading dearth of heavy ions. Thus, some care in designing strategies for data acquisition is necessary. For example, spectra should be acquired several times with different combinations of gates at different $m / z$ values to discern whether ions at high mass are being sampled properly.

\section{Ion Association Maps}

The observed associations are plotted on maps such as those in Figure 6. The lines connect the formulas of ions that were found to be associated. For example, Figure 6a shows that $\mathrm{PO}^{+}$was associated with $\mathrm{V}^{+}$, $\mathrm{VO}^{+}, \mathrm{VO}_{2}^{+}$, and $\mathrm{VPO}_{3}^{+}$. Listed ions that lack connecting lines were observed in the spectra but were not found to associate. Ions crossed out were not observed from the particular compound studied. Ions labeled with an asterisk were deliberately omitted from observation by using a gate that prevented them from being recorded by the TDC. Formulas in parentheses represent tentative assignments.

As with the spectra reported above, the use of gates affected the observed associations. Typical behavior is illustrated by comparing the three maps in Figure 6 . In the situation depicted in Figure 6a, no gates were used, and associations were seen only for relatively light ions. The light ions were ignored in Figure 6b, as indicated by the asterisks on $\mathrm{PO}^{+}, \mathrm{V}^{+}, \mathrm{PO}_{2}^{+}$, and $\mathrm{VO}$, and $\mathrm{VO}_{2}$. When these ions were ignored, several associations involving heavier ions (e.g., $\mathrm{V}_{2} \mathrm{O}_{6}^{+}$ with $\mathrm{V}_{2} \mathrm{O}_{3}^{+}$) appeared. (VO) ${ }_{2} \mathrm{P}_{2} \mathrm{O}_{7}$ was analyzed repeatedly with various combinations of gates. All the resulting associations from these experiments are compiled and depicted together in the net map of Figure $6 \mathrm{c}$. This latter map shows that most ions observed from (VO) ${ }_{2} \mathrm{P}_{2} \mathrm{O}_{7}$ were actually associated with at least one other ion. An interesting exception is $\mathrm{PO}_{2}^{+}$, which was fairly abundant (peak no. 4 in Figure 3a) but was not associated with any other ions. The use of gates had similar, but less dramatic, effects on the observed associations for negative ions from $(\mathrm{VO})_{2} \mathrm{P}_{2} \mathrm{O}_{7}$, as shown in Figure 7.

The ion association maps shown in Figures 8-10 are net maps like those of Figures $6 c$ and 7c. Such a net map is shown for positive ions from unsubstituted $\beta-\mathrm{VOPO}_{4}$ in Figure 8a. Only three associations were seen for unsubstituted $\beta-\mathrm{VOPO}_{4}$, far fewer than the 42 associations found for $(\mathrm{VO})_{2} \mathrm{P}_{2} \mathrm{O}_{7}$ (Figure 6c). Partial substitution of ${ }^{18} \mathrm{O}$ into $\beta-\mathrm{VOPO}_{4}$ induced numerous associations (Figure $8 b$ ). Several ions from substituted $\beta-\mathrm{VOPO}_{4}$, for example, $\mathrm{V}_{2} \mathrm{O}_{4}^{+}, \mathrm{VPO}_{3}^{+}$, and $\mathrm{V}_{2} \mathrm{O}_{3}^{+}$, were found to be associated with their own isotope peaks, indicating that at least two of these ions were created consistently in the same laser pulse. Similar trends were seen for associations between negative ions from $\beta-\mathrm{VOPO}_{4}$ and ${ }^{18} \mathrm{O}$-substituted $\beta-\mathrm{VOPO}_{4}$ (Figure 9).

The few associations seen for $\beta$-VOPO ${ }_{4}$, compared with the numerous ones for ${ }^{18} \mathrm{O}$-substituted $\beta-\mathrm{VOPO}_{4}$ or $(\mathrm{VO})_{2} \mathrm{P}_{2} \mathrm{O}_{7}$, invite comment. These compounds were not that different in structure, bonding, or stoichiometry, and the averaged spectra from them (Figures 3-5) did not differ greatly. Yet the observed numbers of associations differed greatly for the three compounds on a consistent basis over numerous experiments. Apparently, substantial differences in the tendencies for association can occur even for closely 

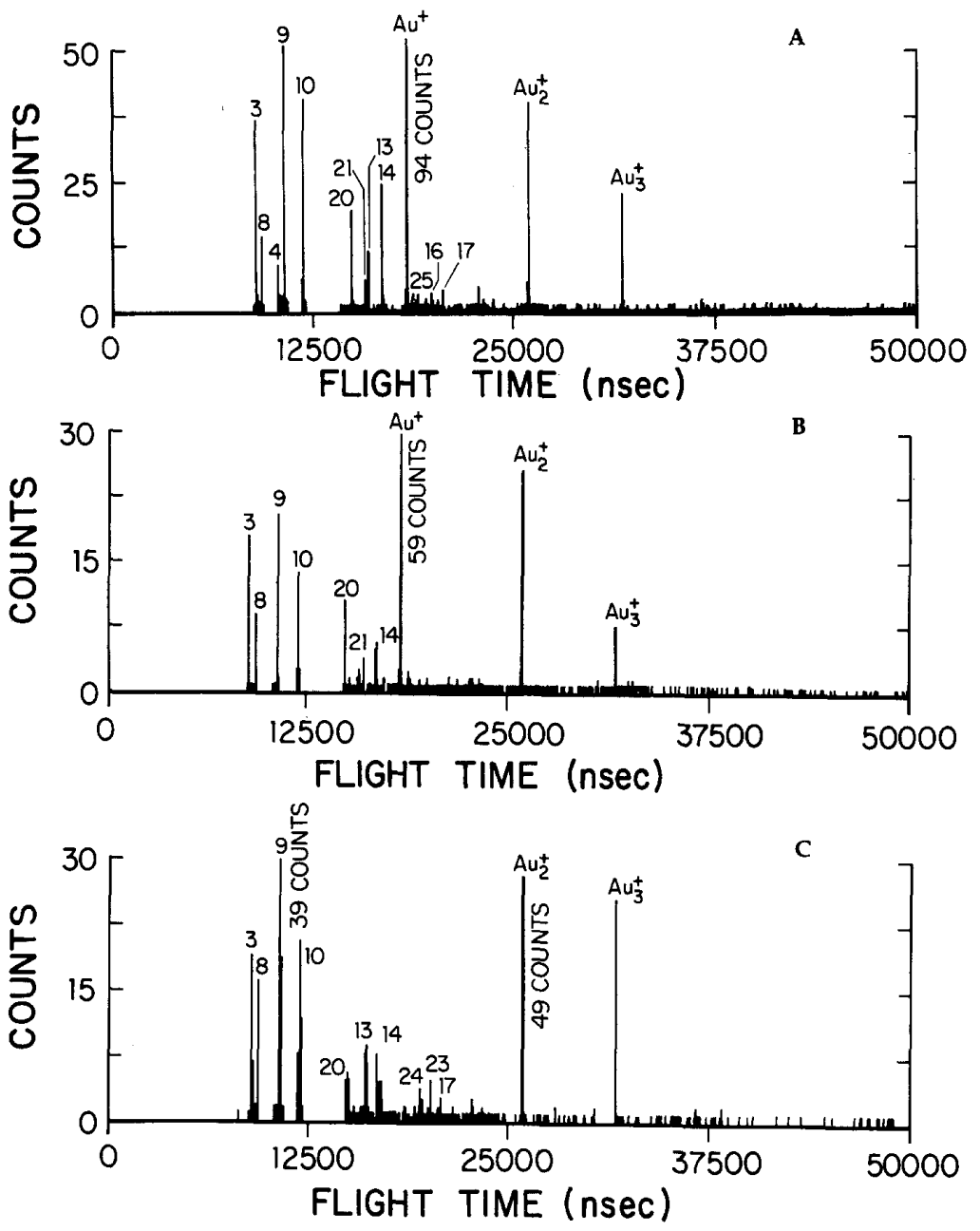

Figure 3. Positive ion spectra of (a) $(\mathrm{VO})_{2} \mathrm{P}_{2} \mathrm{O}_{7}$, (b) $\beta$-VOPO ${ }_{4}$, and (c) ${ }^{18} \mathrm{O}$-substituted $\beta$-VOPO ${ }_{4}$.
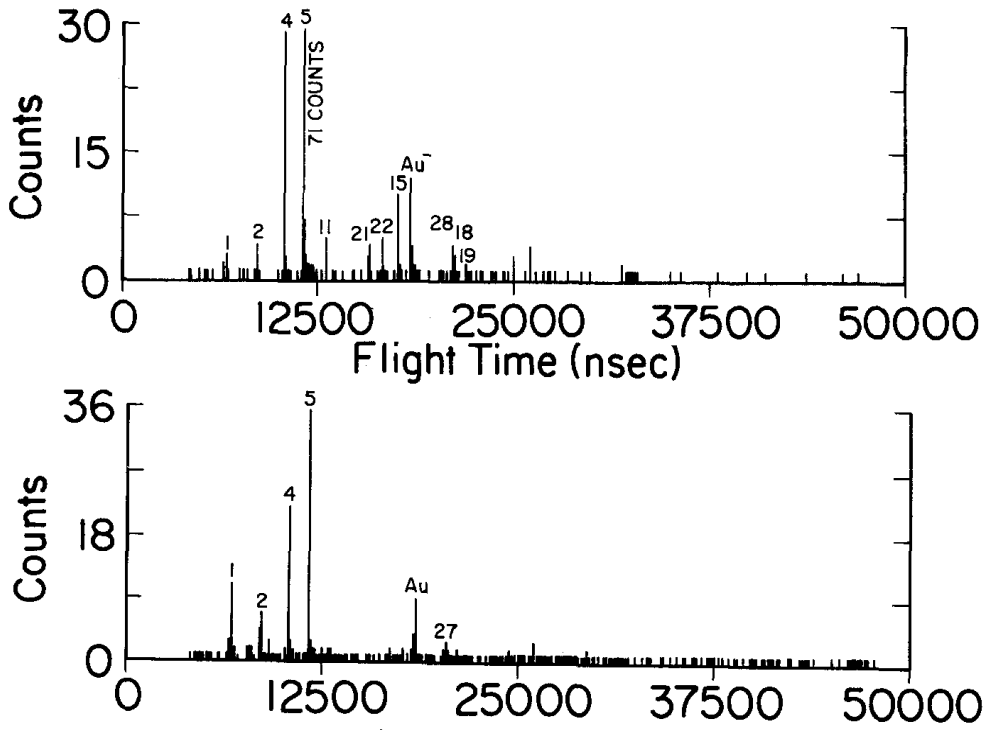

Flight Time (nsec)

Figure 4. Negative ion spectra of (a) $(\mathrm{VO})_{2} \mathrm{P}_{2} \mathrm{O}_{7}$ and (b) $\beta$-VOPO ${ }_{4}$. 
Figure 5. Negative ion spectra of ${ }^{18} \mathrm{O}$-substituted $\beta$-VOPO ${ }_{4}$. (a) No gates used; (b) gate used to ignore ions below $\sim 12,000$ ns. Note greater abundances of heavier ions when gate was used (b).
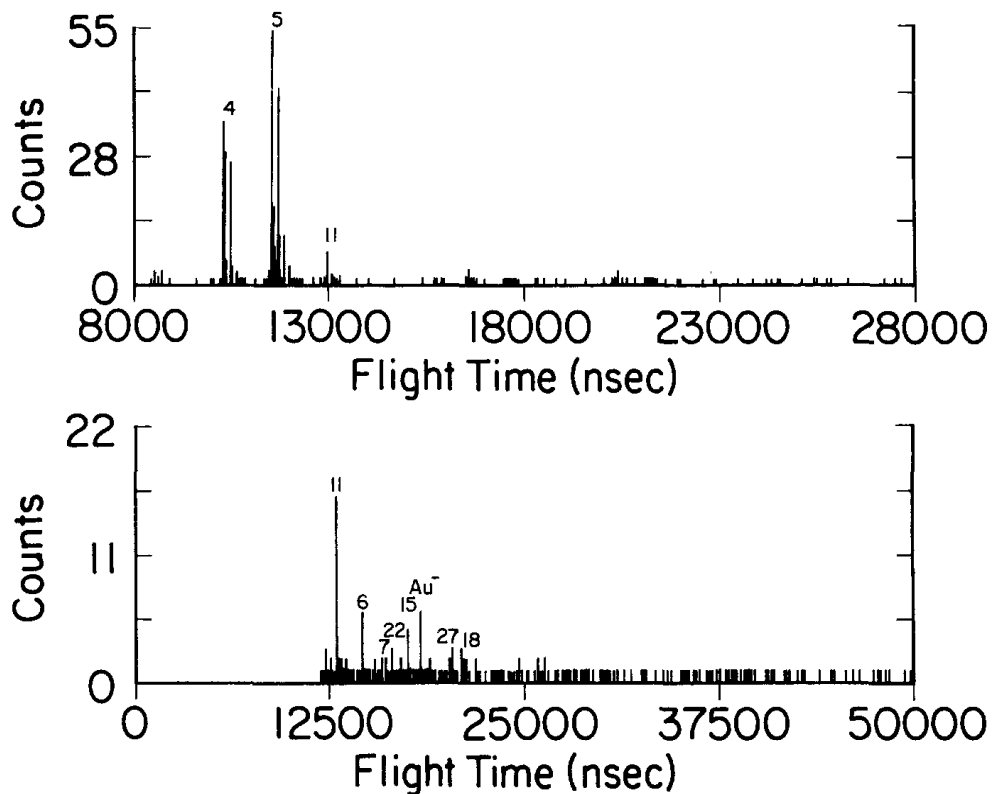

Table 2. Assignments of ions observed

\begin{tabular}{|c|c|c|c|c|c|c|c|c|}
\hline \multirow[b]{2}{*}{ No. ${ }^{a}$} & \multirow[b]{2}{*}{$\begin{array}{l}\text { Assigned } \\
\text { formula }\end{array}$} & \multirow[b]{2}{*}{$m / z$} & \multicolumn{3}{|c|}{ Positive ions } & \multicolumn{3}{|c|}{ Negative ions } \\
\hline & & & $\begin{array}{l}\hat{O}^{N} \\
0^{N} \\
\stackrel{0}{0} \\
\geq\end{array}$ & $\begin{array}{l}0^{ \pm} \\
0 \\
0 \\
01 \\
0\end{array}$ & 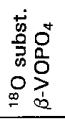 & $\begin{array}{l}0 \\
0 \\
0^{n} \\
0 \\
\geq\end{array}$ & $\begin{array}{l}0 \\
0 \\
0 \\
0 \\
01 \\
0\end{array}$ & 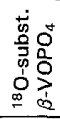 \\
\hline 1 & $(\mathrm{CN})$ & 26 & & & & $x$ & $x$ & $x$ \\
\hline 2 & (HOCN) & 43 & & & & $x$ & $x$ & $x$ \\
\hline 3 & $\mathrm{PO}$ & 47 & $x$ & $x$ & $x$ & & & \\
\hline 4 & $\mathrm{PO}_{2}$ & 63 & $(X)$ & $(X)$ & $(X)$ & $x$ & $x$ & $x$ \\
\hline 5 & $\mathrm{PO}_{3}$ & 79 & & & & $x$ & $x$ & $x$ \\
\hline 6 & $\left(\mathrm{P}_{2} \mathrm{O}_{4}\right)$ & 126 & & & & & $x$ & \\
\hline 7 & $\left(\mathrm{HP}_{2} \mathrm{O}_{6}\right)$ & 159 & & & & & $x$ & $x$ \\
\hline 8 & V & 51 & $x$ & $x$ & $x$ & & & \\
\hline 9 & vo & 67 & $x$ & $x$ & $x$ & & & \\
\hline 10 & $\mathrm{VO}_{2}$ & 83 & $x$ & $x$ & $x$ & & & \\
\hline 11 & $\mathrm{VO}_{3}$ & 99 & & & & $x$ & $x$ & $x$ \\
\hline 12 & $\mathrm{~V}_{2} \mathrm{O}_{2}$ & 134 & $x$ & $x$ & $x$ & & & \\
\hline 13 & $\mathrm{~V}_{2} \mathrm{O}_{3}$ & 150 & $x$ & $x$ & $x$ & & & \\
\hline 14 & $\mathrm{~V}_{2} \mathrm{O}_{4}$ & 166 & $x$ & $x$ & $x$ & & & \\
\hline 15 & $\mathrm{~V}_{2} \mathrm{O}_{5}$ & 182 & & & & $x$ & $x$ & $x$ \\
\hline 16 & $\mathrm{~V}_{3} \mathrm{O}_{5}$ & 233 & $x$ & & $x$ & & & \\
\hline 17 & $\mathrm{~V}_{3} \mathrm{O}_{6}$ & 249 & $x$ & & $x$ & & & $(X)$ \\
\hline 18 & $\mathrm{~V}_{3} \mathrm{O}_{7}$ & 265 & & & & $x$ & $x$ & $x$ \\
\hline 19 & $\mathrm{~V}_{3} \mathrm{O}_{8}$ & 281 & & & & $x$ & $(X)$ & \\
\hline 20 & $\mathrm{VPO}_{3}$ & 130 & $x$ & $x$ & $x$ & & & \\
\hline 21 & $\mathrm{VPO}_{4}$ & 146 & $x$ & $(X)$ & $x$ & $x$ & $x$ & $x$ \\
\hline 22 & $\mathrm{VPO}_{5}$ & 162 & & & & $x$ & $x$ & $x$ \\
\hline 23 & $\mathrm{VP}_{2} \mathrm{O}_{8}$ & 241 & & & $(X)$ & & $x$ & $x$ \\
\hline 24 & $V P_{3} O_{5}$ & 224 & $(X)$ & $(X)$ & $x$ & & & \\
\hline 25 & $\mathrm{~V}_{2} \mathrm{PO}_{5}$ & 213 & $x$ & & $x$ & & & \\
\hline 26 & $\mathrm{~V}_{2} \mathrm{PO}_{6}$ & 229 & $(X)$ & & & (X) & & \\
\hline 27 & $\mathrm{~V}_{2} \mathrm{PO}_{7}$ & 245 & & & & $x$ & $x$ & $x$ \\
\hline 28 & $\mathrm{~V}_{2} \mathrm{PO}_{8}$ & 261 & & & & $x$ & $x$ & $x$ \\
\hline
\end{tabular}


B

A
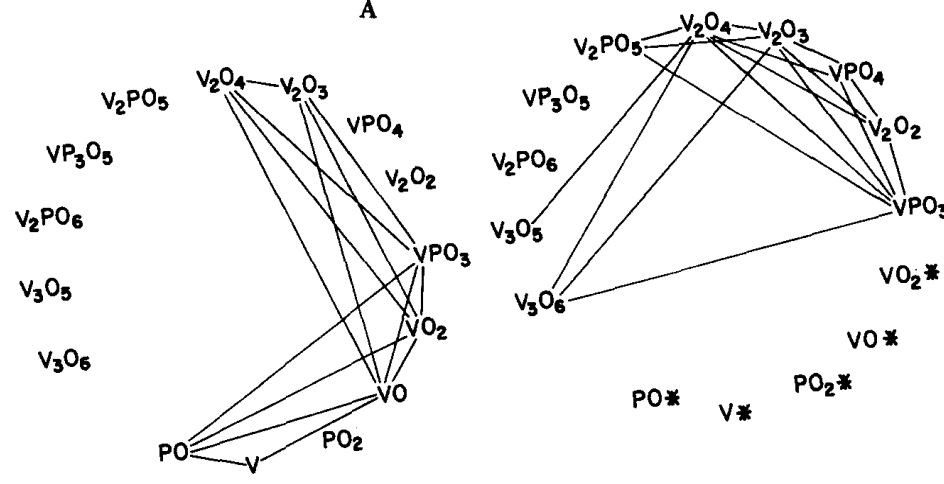

C

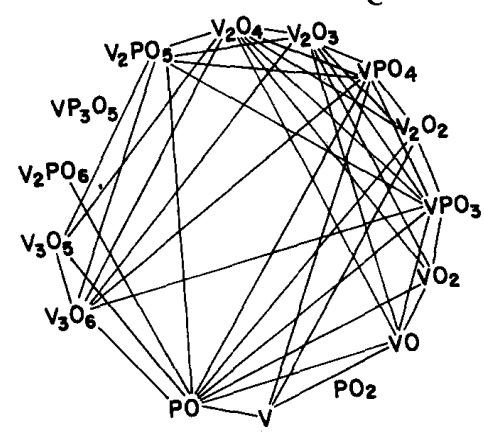

A

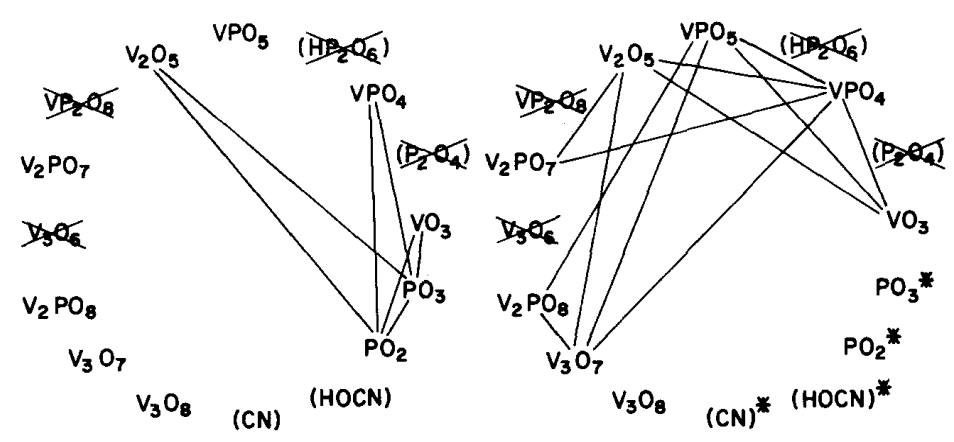

C

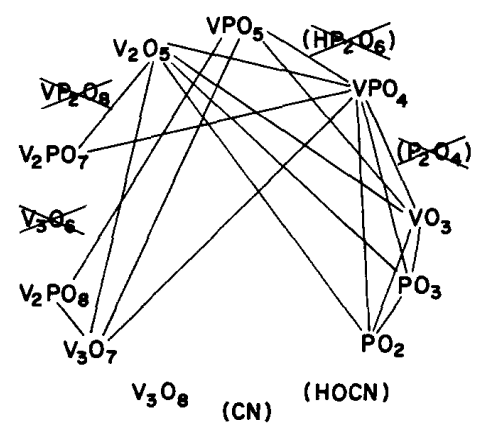

B
Figure 6. Association maps for positive ions from (VO) ${ }_{2} \mathrm{P}_{2} \mathrm{O}_{7}$. (a) No gates used; (b) gate set to ignore ions labeled with asterisks; (c) net map showing all associations combined from use of various gates. 
Figure 8. Net association maps for positive ions from (a) $\beta$-VOPO and (b) ${ }^{18} \mathrm{O}$-substituted $\beta$-VOPO${ }_{4}$. In (b) a horizontal line over a formula indicates that this ion was associated with its own isotope peak.
Figure 9. Net association maps for negative ions from (a) $\beta$-VOPO and (b) ${ }^{18} \mathrm{O}$-substituted $\beta$-VOPO ${ }_{4}$.
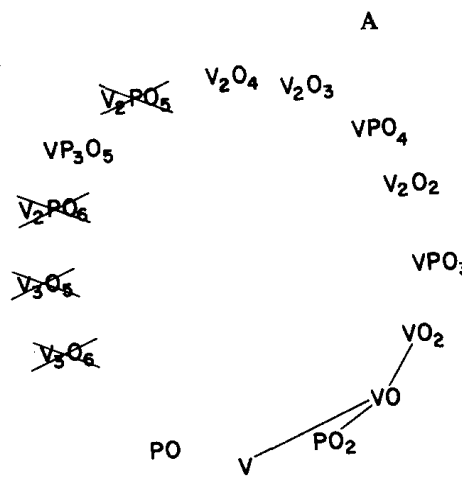

A

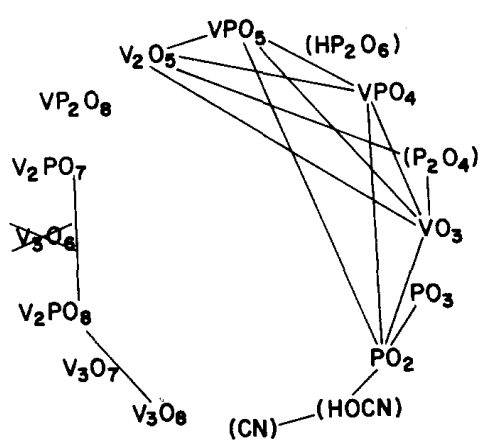

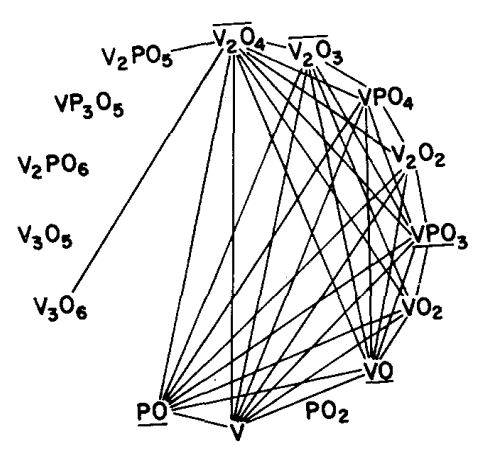

B

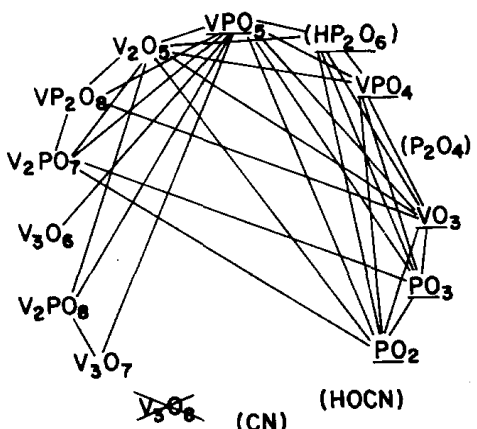

related compounds whose overall spectra are quite similar. Such tendencies are lost in averaged spectra. Thus, storage and subsequent examination of individual scans to discern association may become of considerable value.

\section{Effect of Multiplicity on Associations}

In this work, multiplicity refers to the number of ions produced per scan [3]. Multiplicity may influence ion

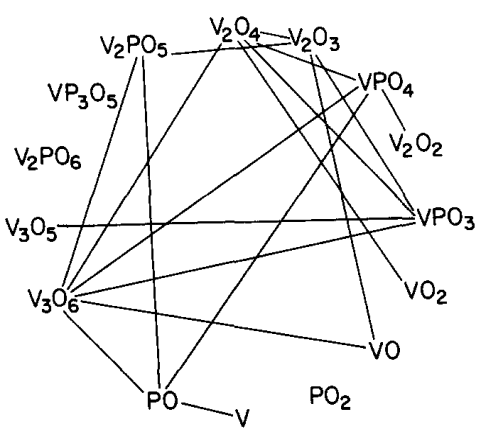

Figure 10. Net association map for positive ions from $(\mathrm{VO})_{2} \mathrm{P}_{2} \mathrm{O}_{7}$ including only those scans when the multiplicity was 7 or less. Compare to Figure 6c. association; for example, the more ions produced per shot, the more likely it is that associations may be found. However, if the multiplicity is too high, ion association may become difficult to determine accurately. To evaluate this effect, associations were recalculated from the same data for the positive ion scans from (VO) ${ }_{2} \mathrm{P}_{2} \mathrm{O}_{7}$ using modified programs that ignored scans containing eight ions. The resulting net association map is depicted in Figure 10. Comparison of this map with the one in Figure $6 \mathrm{c}$ shows that ignoring scans with eight ions yielded fewer associations, particularly among the lighter ions such as $\mathrm{V}^{+}, \mathrm{VO}^{+}, \mathrm{VO}_{2}^{+}$, and $\mathrm{VPO}_{3}^{+}$. This observation suggested that the scans with eight or more ions contained numerous ions at low mass. Such scans may have resulted from relatively high power laser shots that produced many ions and caused substantial fragmentation. When scans containing eight ions were ignored, two new associations ( $\mathrm{VO}^{+}$with $\mathrm{V}_{3} \mathrm{O}_{6}^{+}$, and $\mathrm{V}_{3} \mathrm{O}_{5}^{+}$ with $\mathrm{VPO}_{3}^{+}$, Figure 10) were observed that were not seen originally (Figure 6c). Both of these new associations involved relatively heavy ions, which were probably produced more efficiently in scans with fewer total ions. Thus, the tendency for a given pair of ions to be associated depends somewhat on the multiplicity, which is clearly an important parameter in ion association studies. 


\section{Acknowledgments}

Ames Laboratory is operated for the U.S. Department of Energy by Iowa State University under contract no. W-7405-Eng82. This research was supported by the Director for Energy Research, Office of Basic Energy Sciences, the Advanced Research and Technology Development (ARTD) Program of the Office of Fossil Energy Research, Morgantown Energy Technology Center (D. Brdar, project leader), and the Materials Preparation Center of the Ames Laboratory. The assistance of Mark Lashier in preparing the samples and in discussing the results is gratefully acknowledged.

\section{References}

1. Ens, W.; Beavis, R.; Bolbach, G.; Main, D.; Schueler, B.; Standing, K. G. Nucl. Instrum. Meth. Phys. Res. 1986, A245, 146.

2. Conzemius, R. J.; Adduci, D.; Huang, L. Q.; Junk, G. A.; Houk, R. S. Int. J. Mass Spectrom. Ion Processes 1989, 90, 281.
3. Huang, L. Q.; Conzemius, R. J.; Junk, G. A.; Houk, R. S. Int. J. Mass Spectrom. Ion Processes 1989, 90, 85.

4. Hodnett, B. K. Catal. Rev.-Sci. Eng. 1985, 27, 373.

5. Centi, G.; Trifiro, F. Chim. Ind. 1986, 68, 74.

6. Centi, G.; Trifiro, F.; Ebner, J. R.; Franchetti, V. M. Chem. Rev. 1988, 88, 55.

7. Lashier, M. E.; Moser, T. P.; Schrader, G. L. In New Developments in Selective Oxidation, Trifiro, F.; Centi, G., Eds.; Elsevier: New York, 1990.

8. Bordes, E. Catal. Today 1987, 1, 499.

9. Moser, T. P.; Schrader, G. L. J. Catal. 1987, 104, 99.

10. Wenig, R. W.; Schrader, G. L. J. Catal. 1986, 92, 612.

11. Pepera, M. A.; Callahan, J. L.; Desmond, M. F.; Milberger, E. C.; Blum, R. R.; Bremer, N. J. J. Am. Chem. Soc. 1985, 107, 4883.

12. Huang, L. Q.; Conzemius, R. J.; Junk, G. A.; Houk, R. S. Anal. Chem. 1988, 60, 1490.

13. Huang, L. Q.; Conzemius, R. J.; Holland, G. E.; Houk, R. S. Anal. Chem. 1988, 60, 1636.

14. Moser, T. P.; Schrader, G. L. J. Catal. 1986, 92, 216.

15. Schoer, J. K. M.S. Dissertation, Iowa State University, Ames, Iowa, 1988. 